\title{
Does Relative Performance of Determinate and Indeterminate Dolichos Bean (Lablab purpureus L. Sweet) recombinant Inbred lines (RILs) Depend on Maturity Duration?
}

\author{
H.R. Uday Kumar ${ }^{1 *}$, M. Byre Gowda ${ }^{2}$ and S. Ramesh ${ }^{1}$ \\ ${ }^{1}$ Department of Genetics and Plant Breeding, University of Agricultural Sciences (UAS), \\ Bengaluru, Karnataka, India \\ ${ }^{2}$ All India Coordinated Research Project on Pigeonpea, UAS, Bengaluru, Karnataka, India \\ *Corresponding author
}

\begin{tabular}{|c|c|}
\hline & A B S T R A C T \\
\hline & The per se performance of two bi-parental crosses-derived determinate and \\
\hline Keywords & indeterminate recombinant inbred lines (RILs) belonging to a range of maturity \\
\hline $\begin{array}{l}\text { Determinate; } \\
\text { Indeterminate; } \\
\text { Maturity groups; } \\
\text { Quantitative traits, } \\
\text { RILs. }\end{array}$ & $\begin{array}{l}\text { to } 50 \% \text { flowering, the HA } 4 \times \text { CPI } 31113 \text { (HACPI } 3 \text { )-derived RILs were classified } \\
\text { into early and medium maturity groups, while HA } 4 \times \text { CPI } 60125 \text { (HACPI 6) } \\
\text { derived RILs were classified into extra early, early, medium and late maturity } \\
\text { groups. The per se performance of determinate and indeterminate RILs of all }\end{array}$ \\
\hline Article Info & maturity groups were comparable for most of the traits. Further, there was lack of \\
\hline $\begin{array}{l}\text { Accepted: } \\
\text { 04 June } 2017 \\
\text { Available Online: } \\
\text { 10 July } 2017\end{array}$ & $\begin{array}{l}\text { group for performance consistency for any of the traits across two years. The } \\
\text { study provided ample evidence for possibility of fixing the loci controlling } \\
\text { economic traits in the genetic background of both determinate and indeterminate } \\
\text { varieties irrespective of their maturity duration. }\end{array}$ \\
\hline
\end{tabular}

\section{Introduction}

The dolichos bean is an under-exploited vegetable-cum-pulse crop widely distributed in many tropical and subtropical countries (Purseglove, 1968; Kay, 1979). More than 150 documented local vernacular names of dolichos bean is a testimony of its world-wide distribution. The importance of dolichos bean as a food crop has been documented in archeo-botanical findings in India prior to 1,500 BC (Fuller, 2003). Presently, dolichos bean is grown in Africa, extending from Cameroon to Swaziland to Zimbabwe through Sudan, Ethiopia, Uganda, Kenya and
Tanzania (Skerman et al., 1991). In South and Central America, East and West Indies, Bangladesh, China and India, dolichos bean is cultivated as annual crop (Whyte et al., 1953). In India, it is predominantly grown in southern districts of Karnataka state and adjoining districts of Tamil Nadu, Andhra Pradesh and Maharashtra. It is predominantly as a rainfed conditions for its fresh immature beans for use as a vegetable (Ayyangar and Nambiar 1935; Shivashankar and Kulkarni, 1989). 
Most cultivars were grown by farmers' display indeterminate growth habit (Ayyangar and Nambiar 1935: Shivashankar and Kulkarni, 1989: Keerthi et al., 2014a). Indeterminacy is advantageous for subsistence production and consumption of dolichos bean, as it enables harvesting of pods in multiple pickings ensuring continuous availability of pods for a longer time (Keerthi et al., 2014a, 2014b, 2016). However, of late, due to market economy there is increased demand for varieties with a determinate growth habit. Determinacy is a plant architectural modification in grain legumes (Huyghe, 1998). The varieties with determinate growth habit exhibit synchronous flowering and maturity and thus enable single harvest of all the pods on a commercial scale, which in-turn facilitates economical transportation of the produce to the markets (Viswantath et al., 1971; Shivashankar and Kulkarni, 1989; Kim et al., 1992).

Determinate types compared to their indeterminate counterparts produce larger number of branches (Adams 1982; Chang et al., 1982; Foley et al., 1986; Singh and Schreoder 1988), exhibit greater economic product yield (EPY) potential (Cober and Tanner 1995) and EPY stability (Kelly et al., 1987; White et al., 1992; Julieret al., 1993a; 1993b; Keerthi et al., 2014b, 2016). Determinates also induce greater allocation of total photosynthates into reproductive growth and sink than their indeterminate counterparts (Huyghe, 1998). Besides these advantages, pods borne by determinates contribute greater photosynthates $(13 \%)$ than those borne by indeterminate varieties (6\%) (Sheoran et al., 1987; Koscielniak et al., 1990; Karivaratharaju and Ramamorthy, 1990). Due to their compact growth, determinates facilitate high density planting to maximize their EPY (Vishwanath et al., 1971; Kim et al., 1992).
Considering the advantages of determinacy, major emphasis of dolichos bean breeding has been to develop determinate varieties. However, growth habit is reported to affect productivity of pod and seed yield per se and their component traits in dolichos bean (Keerthi et al., 2014a). There have been numerous reports on the effect of growth habit on productivity of pod and seed yield and their component traits soybean (Bernard 1972; Cooper and Waranyawat 1985; Parvez et al., 1989; Wilcox and Zhang 1997; Robinson and Wilcox 1998). Most of these reports indicate superiority of indeterminate genotypes over determinate counterparts for seed yield.

Nlaya et al., (1999) reported higher yielding ability of six indeterminate cultivars than that of determinate pinto bean (Phaseolus vulgaris) cultivars under available soil moisture gradient in dry-land conditions. In fababean, Nadal et al., (2005) documented higher dry seed yield of the three indeterminate cultivars than that of three determinate cultivars.

In dolichos bean, Keerthi et al., (2014) based on a random sample of unrelated determinate and indeterminate genotypes opined that performance stability of determinate genotypes was better than that of their indeterminate counterparts. However, these studies are based on limited number of genotypes with a particular maturity group. Considering that crop performance is directly related to duration, any such comparative performance studies should be based on determinate and indeterminate genotypes belonging to a range of maturity groups. The objective of the present investigation was to compare the pod and seed yield and their component traits between the determinate and indeterminate recombinant inbreed lines (RILs) belonging to a range of maturity groups in dolichos bean. 


\section{Materials and Methods}

The material for the study comprised of 157 RILs derived from HA $4 \times$ CPI 31113 (here after referred as HACPI 3) and 144 RILs derived from HA $4 \times$ CPI 60125(here after referred as HACPI 6) and three check entries [HA 3, HA 4 and kadalavare (KA)] maintained at All India Co-ordinated Research Project (AICRP) on pigeonpea, University of Agricultural Sciences (UAS), Bengaluru, India. The parents of RILs, HA 4, CPI 31113 and CPI 60125 differs from for fresh pod yield and its component traits such as number of racemes, raceme length, fresh pods raceme ${ }^{-1}$, and fresh pods plant ${ }^{-1}$. The seedlings of all the RILs and the checks were raised in polythene covers and maintained for 15-20 days for proper rooting. Subsequently, the seedlings of two RIL populations and those of the three check entries were transplanted to field in an augmented design (Federer 1956) in eight compact blocks for each RIL population during 2014 and 2015 rainy seasons at the experimental plot of Zonal Agricultural Research Station (ZARS), UAS, Bengaluru. Each block consisted of 1820 RILs, three checks and two border entries. The seedlings of each entry were transplanted in a single row of $2.5 \mathrm{~m}$ length, with a row spacing of $0.45 \mathrm{~m}$. A basal dose of 25:50:25 $\mathrm{Kg} \mathrm{ha}{ }^{-1}$ of NPK (nitrogen: phosphorous: potassium) was applied to the experimental plots. Recommended management practices were followed during the crop-growing period to raise a healthy crop.

\section{Sampling of plants and data collection}

In HACPI 3- derived RILs, out of 157 planted, only 136 individuals (66 determinate and 70 indeterminate types) and in HACPI 6derived RILs, out of 144 planted, only 119 individuals (33 determinate and 86 indeterminate types) survived till the maturity. Data were recorded on survived
RILs on eight quantitative traits (QTs) (days to $50 \%$ flowering, raceme bearing branches plant $^{-1}$, raceme length, racemes plant ${ }^{-1}$, fresh pods plant ${ }^{-1}$, fresh pod yield plant ${ }^{-1}$, fresh seed yield plant ${ }^{-1}$ and dry seed yield plant ${ }^{-1}$ ) based on counting/measurement using appropriate scale depending on the trait in each RILs and check entries following the descriptors (Byregowda et al., 2015).

As is true in most grain legumes, in dolichos bean also, the period from days to flowering to days to maturity is by and large remain constant. Taking cue from this, based on days to 50\% flowering, the HACPI 3- derived RILs were classified into early maturity (50-65 days to $50 \%$ flowering) and medium maturity (66-80 days to $50 \%$ flowering), while HACPI 6- derived RILs were classified into four maturity groups such as extra early (40-50 days to $50 \%$ flowering), early (51-60 days to $50 \%$ flowering), medium (61-75 days to $50 \%$ flowering) and late (76-90 days to 50\% flowering).

\section{Statistical analysis}

Pooled analysis of variance was carried out to detect the block $\times$ year, checks $\times$ year, RILs $\times$ year, determinate $x$ years, indeterminate $x$ years and determinatex indeterminate interactions by using Residual Maximum Likelihood (REML) linear mixed model approach (Patterson and Thompson, 1971) implemented using PROC GLM in SAS 9.4 (SAS Institute Inc., Cary, NC, USA). The means of each RILs and each check for all the eight QTs were estimated. Significance of differences in QTs means between determinate and indeterminate RILs derived from HACPI3 and HACPI 6 in each maturity group was examined using two sample t-test assuming unequal variances as number of determinate and indeterminate RILs varied in each maturity group. The test statistic ' $t$ ' was computed as, 
$t=\frac{\left(\bar{X}_{D}-\bar{X}_{I D}\right)}{\sqrt{s p 2}\left(\frac{1}{N_{D}}-\frac{1}{N_{I D}}\right)}$

Where, $\overline{\mathrm{X}}{ }_{\mathrm{D}}=\mathrm{QTs}$ mean in determinate RILs; $\bar{X}{ }_{I D}=$ QTs mean in indeterminate RILs; $\mathrm{sp}^{2}=\left(\mathrm{n}_{1}-1\right) \mathrm{s}_{1}{ }^{2}+\left(\mathrm{n}_{2}-1\right) \mathrm{s}_{2}{ }^{2} / \mathrm{n}_{1}+\mathrm{n}_{2}-2 ; \mathrm{n}_{1}=$ number of determinate RILs; $\mathrm{n}_{2}=$ number of indeterminate RILs; $\mathrm{s}_{1}{ }^{2}=$ variance of determinate RILs; $\mathrm{s}_{2}{ }^{2}=$ variance of indeterminate RILs; $\mathrm{N}_{D}=$ number of determinate RILs; $\mathrm{N}_{\mathrm{ID}}=$ number of indeterminate RILs.

For reliable and unambiguous performance comparison, the trait variances should be homogenous between determinate and indeterminate RILs of each maturity group. Traits phenotypic variances within the determinate and indeterminate RILs of each maturity groups were estimated using 'statistical analysis' option available in Microsoft excel. Homogeneity of traits phenotypic variances between determinate and indeterminate RILs was examined using Levene's test implemented using 'PROC Univariate' (SAS Institute, Cary, NC). To assess the consistency of performance of determinate and indeterminate RILs across two years of their evaluation, spearman rank correlation coefficient $\left(\mathrm{R}_{\mathrm{S}}\right)$ was estimated. To compute $\mathrm{R}_{\mathrm{S}}$, the trait means of each maturity groups of RILs evaluated during 2014 and 2015 were ranked separately and sum of the squared difference between the ranks were computed. $\mathrm{R}_{\mathrm{S}}$ was calculated using the following formula:

$R_{s}=\frac{1-6\left(\sum d^{2}+C F\right)}{n\left(n^{2}-1\right)}$

Where, $d^{2}=$ squared differences between ranks of each RILs evaluated in 2014 and 2015

$\mathrm{CF}=\Sigma\left\{\left(t^{3}-t\right) / 12\right\}$ with ' $\mathrm{t}$ ' being the order of each tie (RILs with same rank) and $n=$ number of RILs.

The significance of $R_{S}$ was examined using Student's $t$ test, as

$t=\frac{R_{s} \sqrt{n-2}}{\sqrt{1-R^{2} s}}$

With $\mathrm{n}-2$ degrees of freedom. If $\mathrm{t} \geq \mathrm{t}(0.01$ or 0.05: $n-2)$, the null hypothesis was discarded and the estimate of ' $R_{S}$ ' was declared as significant.

\section{Results and Discussion}

REML analysis revealed highly significant mean squares attributable to 'determinate RILs', 'indeterminate RILs' and 'checks' for all the eight QTs in both the RIL populations (Results are not provided). These results suggested significant differences among the determinate RILs, indeterminate RILs, between determinate, indeterminate RILs and checks, respectively. Mean squares attributable to checks vs. years were significant for all traits, except days to $50 \%$ flowering and fresh pod plant ${ }^{-1}$ in RILs derived from both the crosses and for fresh seed yield plant ${ }^{-1}$ in HACPI 3-derived RILs. The determinate RILs derived from both the crosses interacted significantly with years for all the QTs except fresh pod yield plant ${ }^{-1}$ and fresh seed yield plant ${ }^{-1}$ in HACPI 3-derived RILs, while indeterminate RILs derived from both the crosses interacted significantly with years for all the QTs except fresh pod yield plant $^{-1}$ in HACPI 3-derived RILs. On the contrary, the determinate RILs interacted significantly with those of indeterminate RILs for all the QTs except fresh seed yield plant ${ }^{-1}$ in HACPI 6- derived RILs. These results indicated differential performance of determinate and indeterminate RILs and checks across two years. 
Non-significance of Levene's test (Tables 1 to 6) indicated homogeneity of QTs variances between determinate and indeterminate RILs of all maturity groups barring a very few exceptions. Such homogeneity of QTs variances is a necessary prerequisite for reliable comparative assessment of determinate and indeterminate RILs of different maturity groups. The HACPI 3derived determinate RILs of early maturity group were significantly early to flower compared to those of indeterminate RILs evaluated during 2014 (Table 8), although the magnitude of differences were marginal to have any practical significance. Similarly, HACPI 6- derived determinate RILs (evaluated during 2015) of early maturity group (Table 11) and those (evaluated during 2014) of medium and late maturity groups (Table 12) were significantly early to flower compared to indeterminate RILs. For fresh and dry seed yield plant ${ }^{-1}$, the two most important economic traits, HACPI 3-derived determinate and indeterminate RILs (evaluated during 2015) of early maturity (Table 8) and those (evaluated during 2014) of medium maturity (Table 12) differed significantly in favour of determinate RILs. However, the magnitude of differences in mean fresh and dry seed yield plant ${ }^{-1}$ were marginal with hardly any practical significance. For rest of the traits in both the years of evaluation, the determinate and indeterminate RILs of all the maturity groups were comparable (Table 8 to 13). By and large, present study indicated comparable per se performance of determinate and indeterminate RILs of different maturity groups for all the traits investigated. Results of this study are in agreement with those of Robinson and Wilcox (1998) who provided evidence that loci affecting superior seed yield expressed in both determinate and indeterminate $\mathrm{F}_{5}$ derived near isogenic lines (NILs) of soybean.

Kato et al., (2015) in a similar effort compared indeterminate and determinate biparental crosses-derived RILs belonging to early, middle and late maturity groups in soybean. They reported non-significant differences in number of pods plant ${ }^{-1}$ and seed weight plant $^{-1}$ between determinate and indeterminate RILs of all the three maturity groups. On the contrary, number of seeds plant $^{-1}$ of indeterminate RILs was more than those of determinate RILs only in early maturity group.

Table.1 Estimates of phenotypic variance within determinate (D) and Indeterminate (ID) early maturity group RILs derived from HACPI 3

\begin{tabular}{|c|c|c|c|c|c|c|}
\hline \multirow[t]{3}{*}{ Traits } & \multirow{2}{*}{\multicolumn{2}{|c|}{$\frac{2014}{\text { Variance }}$}} & \multirow{3}{*}{$\begin{array}{l}\text { Levene } \\
\text { Statisti } \\
\text { c }\end{array}$} & \multicolumn{3}{|c|}{2015} \\
\hline & & & & \multicolumn{2}{|c|}{ Variance } & \multirow{2}{*}{$\begin{array}{c}\text { Levene } \\
\text { Statisti } \\
\text { c }\end{array}$} \\
\hline & $\mathbf{D}$ & ID & & D & ID & \\
\hline Days to $50 \%$ flowering & 6.25 & 2.56 & $\begin{array}{c}15.99 * \\
*\end{array}$ & 19.60 & 15.85 & 1.56 \\
\hline Raceme bearing branches plant $^{-1}$ & 15.40 & 56.47 & $4.16^{*}$ & 8.83 & 10.70 & 0.26 \\
\hline Raceme length $(\mathrm{cm})$ & 22.93 & 14.77 & 1.18 & 4.03 & 2.29 & 2.15 \\
\hline Racemes plant $^{-1}$ & 3.45 & 4.60 & 0.33 & 1.26 & 1.63 & 0.20 \\
\hline Fresh pods plant $^{-1}$ & 130.12 & 71.32 & 3.47 & 111.51 & 43.67 & $5.11 *$ \\
\hline Fresh pod yield plant $^{-1}(\mathrm{~g})$ & 510.75 & 391.04 & 0.15 & 541.24 & 149.35 & $4.46^{*}$ \\
\hline Fresh seed yield plant $^{-1}(\mathrm{~g})$ & 124.53 & 76.57 & 0.05 & 60.69 & 32.88 & 2.92 \\
\hline Dry seed yield plant $^{-1}(\mathrm{~g})$ & 23.80 & 64.28 & 3.71 & 19.11 & 6.96 & $3.80 *$ \\
\hline
\end{tabular}

* Significant at $\mathrm{P}=0.05 ; * *$ Significant at $\mathrm{P}=0.01$ 
Table.2 Estimates of phenotypic variance within determinate (D) and indeterminate (ID) Medium maturity group RILs derived from HACPI 3

\begin{tabular}{|c|c|c|c|c|c|c|}
\hline \multirow[t]{3}{*}{ Traits } & \multirow{2}{*}{\multicolumn{2}{|c|}{$\begin{array}{c}2014 \\
\text { Variance }\end{array}$}} & \multirow{3}{*}{$\begin{array}{l}\text { Levene } \\
\text { Statistic }\end{array}$} & \multicolumn{3}{|c|}{2015} \\
\hline & & & & \multicolumn{2}{|c|}{ Variance } & \multirow{2}{*}{$\begin{array}{c}\text { Levene } \\
\text { Statistic }\end{array}$} \\
\hline & $\mathbf{D}$ & ID & & $\mathbf{D}$ & ID & \\
\hline Days to $50 \%$ flowering & 21.19 & 21.58 & 0.19 & 5.27 & 5.91 & 0.48 \\
\hline Raceme bearing branches plant $^{-1}$ & 17.26 & 24.17 & 0.43 & 8.90 & 9.61 & 0.69 \\
\hline Raceme length $(\mathrm{cm})$ & 12.86 & 4.61 & $10.94 * *$ & 8.52 & 3.09 & $6.14 * *$ \\
\hline Racemes plant $^{-1}$ & 2.64 & 1.25 & 2.27 & 2.09 & 1.90 & 0.32 \\
\hline Fresh pods plant $^{-1}$ & 278.23 & 76.69 & 5.51 & 113.51 & 81.00 & 1.68 \\
\hline Fresh pod yield plant $^{-1}(\mathrm{~g})$ & 702.90 & 206.89 & $9.10 *$ & 206.68 & 389.82 & 0.02 \\
\hline Fresh seed yield plant $^{-1}(\mathrm{~g})$ & 165.81 & 23.97 & $12.87 * *$ & 66.77 & 111.05 & 0.06 \\
\hline Dry seed yield plant $^{-1}(\mathrm{~g})$ & 58.47 & 16.57 & $3.91 *$ & 11.49 & 19.42 & 0.02 \\
\hline
\end{tabular}

* Significant at $\mathrm{P}=0.05 ; * *$ Significant at $\mathrm{P}=0.01$

Table.3 Estimates of phenotypic variance within determinate (D) and indeterminate (ID) Extra early maturity group RILs derived from HACPI 6

\begin{tabular}{|c|c|c|c|c|c|c|}
\hline \multirow[t]{3}{*}{ Traits } & \multirow{2}{*}{\multicolumn{2}{|c|}{\begin{tabular}{|c|}
2014 \\
Variance
\end{tabular}}} & \multirow{3}{*}{$\begin{array}{l}\text { Levene } \\
\text { Statistic }\end{array}$} & \multicolumn{3}{|c|}{2015} \\
\hline & & & & \multicolumn{2}{|c|}{ Variance } & \multirow{2}{*}{$\begin{array}{l}\text { Levene } \\
\text { Statistic }\end{array}$} \\
\hline & D & ID & & $\mathbf{D}$ & ID & \\
\hline Days to $50 \%$ flowering & 7.73 & 9.55 & 1.91 & 9.86 & 2.62 & $5.39 *$ \\
\hline Raceme bearing branches plant $^{-1}$ & 12.01 & 23.93 & 1.30 & 6.37 & 5.15 & 0.32 \\
\hline Raceme length $(\mathrm{cm})$ & 4.61 & 4.91 & 0.01 & 1.96 & 1.56 & 0.00 \\
\hline Racemes plant $^{-1}$ & 2.19 & 2.52 & 0.80 & 0.93 & 0.81 & 0.28 \\
\hline Fresh pods plant $^{-1}$ & 73.60 & 46.39 & 0.01 & 48.35 & 29.11 & 0.78 \\
\hline Fresh pod yield plant $^{-1}(\mathrm{~g})$ & 196.30 & 86.50 & 0.85 & 162.88 & 93.10 & 0.62 \\
\hline Fresh seed yield plant $^{-1}(\mathrm{~g})$ & 19.98 & 57.37 & 0.54 & 32.62 & 30.71 & 0.02 \\
\hline Dry seed yield plant $^{-1}(\mathrm{~g})$ & 7.71 & 29.42 & $5.93^{*}$ & 6.88 & 9.42 & 0.38 \\
\hline
\end{tabular}

* Significant at $\mathrm{P}=0.05 ; * *$ Significant at $\mathrm{P}=0.01$

Table.4 Estimates of phenotypic variance within determinate (D) and indeterminate (ID) Early maturity group RILs derived from HACPI 6

\begin{tabular}{|c|c|c|c|c|c|c|}
\hline \multirow{3}{*}{ Traits } & \multirow{2}{*}{\multicolumn{2}{|c|}{$\begin{array}{c}2014 \\
\text { Variance } \\
\end{array}$}} & \multirow{3}{*}{$\begin{array}{l}\text { Levene } \\
\text { Statistic }\end{array}$} & \multicolumn{3}{|c|}{2015} \\
\hline & & & & \multicolumn{2}{|c|}{ Variance } & \multirow{2}{*}{$\begin{array}{l}\text { Levene } \\
\text { Statistic }\end{array}$} \\
\hline & $\mathbf{D}$ & ID & & D & ID & \\
\hline Days to $50 \%$ flowering & 2.25 & 6.07 & $15.07 * *$ & 5.00 & 7.11 & $4.85 *$ \\
\hline Raceme bearing branches plant $^{-1}$ & 1.48 & 13.09 & 1.07 & 7.31 & 11.44 & 2.02 \\
\hline Raceme length (cm) & 14.43 & 5.75 & 0.80 & 1.44 & 1.94 & 0.92 \\
\hline Racemes plant $^{-1}$ & 5.37 & 2.90 & 0.40 & 0.91 & 0.85 & 0.18 \\
\hline Fresh pods plant $^{-1}$ & 57.00 & 34.46 & 0.08 & 12.30 & 57.69 & 2.49 \\
\hline Fresh pod yield plant $^{-1}(\mathrm{~g})$ & 19.80 & 55.96 & 1.17 & 44.45 & 183.85 & 2.86 \\
\hline Fresh seed yield plant $^{-1}(\mathrm{~g})$ & 1.19 & 17.33 & 3.54 & 7.34 & 41.47 & 3.46 \\
\hline Dry seed yield plant $^{-1}(\mathrm{~g})$ & 3.90 & 5.89 & 0.20 & 2.84 & 7.29 & 1.63 \\
\hline
\end{tabular}

* Significant at $\mathrm{P}=0.05$; ** Significant at $\mathrm{P}=0.01$ 
Table.5 Estimates of phenotypic variance within determinate (D) and indeterminate (ID) Medium maturity group RILs derived from HACPI 6

\begin{tabular}{|c|c|c|c|c|c|c|}
\hline \multirow[t]{3}{*}{ Traits } & \multirow{2}{*}{\multicolumn{2}{|c|}{\begin{tabular}{|c|}
2014 \\
Variance
\end{tabular}}} & \multirow{3}{*}{$\begin{array}{l}\text { Levene } \\
\text { Statistic }\end{array}$} & \multicolumn{3}{|c|}{2015} \\
\hline & & & & \multicolumn{2}{|c|}{ Variance } & \multirow{2}{*}{$\begin{array}{l}\text { Levene } \\
\text { Statistic }\end{array}$} \\
\hline & $\mathbf{D}$ & ID & & $\mathbf{D}$ & ID & \\
\hline Days to $50 \%$ flowering & 6.43 & 6.84 & $11.50 * *$ & 19.55 & 11.41 & 0.73 \\
\hline Raceme bearing branches plant ${ }^{-1}$ & 30.64 & 18.59 & 0.58 & 20.18 & 6.89 & $6.92 * *$ \\
\hline Raceme length $(\mathrm{cm})$ & 7.52 & 9.51 & 0.44 & 1.30 & 11.97 & 0.29 \\
\hline Racemes plant $^{-1}$ & 3.06 & 3.85 & 0.40 & 0.64 & 1.48 & 2.39 \\
\hline Fresh pods plant $^{-1}$ & 62.70 & 74.77 & 0.01 & 53.27 & 55.05 & 0.14 \\
\hline Fresh pod yield plant $^{-1}(\mathrm{~g})$ & 72.57 & 179.06 & $5.16^{*}$ & 94.43 & 116.33 & 0.08 \\
\hline Fresh seed yield plant $^{-1}(\mathrm{~g})$ & 16.59 & 45.97 & $3.92 *$ & 25.77 & 27.45 & 0.01 \\
\hline Dry seed yield plant $^{-1}(\mathrm{~g})$ & 9.43 & 20.62 & 1.50 & 5.57 & 8.58 & 0.68 \\
\hline
\end{tabular}

* Significant at $\mathrm{P}=0.05 ; * *$ Significant at $\mathrm{P}=0.01$

Table.6 Estimates of phenotypic variance within determinate (D) and indeterminate (ID) Late maturity group RILs derived from HACPI 6

\begin{tabular}{|c|c|c|c|c|c|c|}
\hline \multirow[t]{3}{*}{ Traits } & \multirow{2}{*}{\multicolumn{2}{|c|}{$\begin{array}{c}2014 \\
\text { Variance }\end{array}$}} & \multirow{3}{*}{$\begin{array}{l}\text { Levene } \\
\text { Statistic }\end{array}$} & \multicolumn{3}{|c|}{2015} \\
\hline & & & & \multicolumn{2}{|c|}{ Variance } & \multirow{2}{*}{$\begin{array}{l}\text { Levene } \\
\text { Statistic }\end{array}$} \\
\hline & $\mathbf{D}$ & ID & & $\mathbf{D}$ & ID & \\
\hline Days to $50 \%$ flowering & 1.33 & 9.76 & 0.88 & 14.92 & 1.21 & $9.72 * *$ \\
\hline Raceme bearing branches plant $^{-1}$ & 4.89 & 30.50 & 1.11 & 4.12 & 2.96 & 0.03 \\
\hline Raceme length $(\mathrm{cm})$ & 4.50 & 9.28 & 1.37 & 1.00 & 0.68 & 0.20 \\
\hline Racemes plant $^{-1}$ & 2.09 & 3.08 & 0.12 & 1.05 & 0.66 & 0.21 \\
\hline Fresh pods plant $^{-1}$ & 50.33 & 105.12 & 0.71 & 161.33 & 75.21 & 0.89 \\
\hline Fresh pod yield plant $^{-1}(\mathrm{~g})$ & 226.16 & 388.57 & 0.01 & 336.88 & 178.61 & 0.34 \\
\hline Fresh seed yield plant $^{-1}(\mathrm{~g})$ & 37.36 & 39.44 & 0.03 & 54.44 & 36.81 & 0.13 \\
\hline Dry seed yield plant $^{-1}(\mathrm{~g})$ & 26.88 & 299.89 & 0.17 & 21.62 & 7.27 & 0.77 \\
\hline
\end{tabular}

* Significant at $\mathrm{P}=0.05 ; * *$ Significant at $\mathrm{P}=0.01$

Table.7 Estimates of rank correlation between quantitative trait means of HACPI 3-dervied RILs evaluated in 2014 and 2015

\begin{tabular}{|l|c|c|c|c|}
\hline \multirow{2}{*}{ Trait } & \multicolumn{2}{c|}{ Early maturity group } & \multicolumn{2}{c|}{ Medium maturity group } \\
\cline { 2 - 5 } & Determinate & Indeterminate & Determinate & Indeterminate \\
\hline Days to 50\% flowering & -0.10 & $0.55^{*}$ & 1.00 & -0.47 \\
\hline Raceme bearing branches plant $^{-1}$ & -0.12 & 0.37 & 0.14 & -0.09 \\
\hline Raceme length (cm) $^{-1}$ & 0.20 & $0.67^{*}$ & 0.48 & -0.06 \\
\hline Racemes plant $^{-1}$ & 0.60 & $0.89^{* *}$ & 0.42 & $0.62^{*}$ \\
\hline Fresh pods plant $^{-1}$ & 0.57 & $0.71^{*}$ & $0.54^{*}$ & -0.05 \\
\hline Fresh pod yield plant $^{-1}(\mathrm{~g})$ & 0.50 & $0.71^{*}$ & $0.60^{*}$ & -0.17 \\
\hline Fresh seed yield plant $^{-1}(\mathrm{~g})$ & $0.90^{*}$ & $0.64^{*}$ & $0.75^{*}$ & -0.15 \\
\hline Dry seed yield plant $^{-1}(\mathrm{~g})$ & 0.60 & 0.45 & $0.75^{*}$ & -0.05 \\
\hline
\end{tabular}

$*=$ Significant at $\mathrm{P}=0.05 * *=$ Significant at $\mathrm{P}=0.01$ 
Table.8 Comparative quantitative trait means of early maturity group (50-65 DAS)

Determinate and indeterminate HACPI 3-derived RILs

\begin{tabular}{|c|c|c|c|c|c|c|c|c|}
\hline & \multicolumn{2}{|c|}{2014} & \multirow[b]{4}{*}{ Difference } & \multirow[b]{4}{*}{ t-statistic } & \multicolumn{2}{|c|}{2015} & \multirow[b]{4}{*}{ Difference } & \multirow[b]{4}{*}{ t-statistic } \\
\hline & Determinate & Indeterminate & & & Determinate & Indeterminate & & \\
\hline Number of RILs $\underset{ }{\longrightarrow}$ & 16 & 26 & & & 19 & 42 & & \\
\hline Trait & & & & & & & & \\
\hline Days to $50 \%$ flowering & 57.88 & 64.35 & 6.47 & $-9.25 * *$ & 57.47 & 59.05 & 1.57 & -1.32 \\
\hline Raceme bearing branches plant $^{-1}$ & 8.96 & 13.23 & 4.27 & $-2.41 * *$ & 14.03 & 14.84 & 0.81 & -0.95 \\
\hline Raceme length $(\mathrm{cm})$ & 14.05 & 11.90 & 2.15 & 1.51 & 11.70 & 11.81 & 0.11 & -0.2 \\
\hline Racemes plant $^{-1}$ & 8.35 & 8.60 & 0.25 & -0.39 & 8.61 & 8.68 & 0.07 & -0.21 \\
\hline Fresh pods plant $^{-1}$ & 27.63 & 21.73 & 5.89 & 1.78 & 33.79 & 29.52 & 4.27 & 1.62 \\
\hline Fresh pod yield plant $^{-1}(\mathrm{~g})$ & 45.55 & 39.69 & 5.86 & 0.85 & 53.18 & 43.03 & 10.15 & 1.79 \\
\hline Fresh seed yield plant $^{-1}(\mathrm{~g})$ & 22.34 & 17.62 & 4.72 & 1.44 & 26.19 & 21.29 & 4.90 & $2.45 * *$ \\
\hline Dry seed yield plant $^{-1}(\mathrm{~g})$ & 11.93 & 11.13 & 0.80 & 0.4 & 13.58 & 10.18 & 3.40 & $3.13 * *$ \\
\hline
\end{tabular}

Table.9 Comparative quantitative trait means of medium maturity group (66-80 DAS)

Determinate and indeterminate HACPI 3-derived RILs

\begin{tabular}{|c|c|c|c|c|c|c|c|c|}
\hline & \multicolumn{2}{|c|}{2014} & \multirow[b]{4}{*}{ Difference } & \multirow[b]{4}{*}{ t-statistic } & \multicolumn{2}{|c|}{2015} & \multirow[b]{4}{*}{ Difference } & \multirow[b]{4}{*}{ t-statistic } \\
\hline & Determinate & Indeterminate & & & Determinate & Indeterminate & & \\
\hline Number of RILs $\Longrightarrow$ & 25 & 13 & & & 19 & 24 & & \\
\hline Trait & & & & & & & & \\
\hline Days to $50 \%$ flowering & 68.76 & 70.08 & 1.32 & -0.83 & 68.95 & 68.50 & 0.45 & 0.61 \\
\hline Raceme bearing branches plant $^{-1}$ & 8.00 & 9.82 & 1.82 & -1.13 & 13.56 & 14.50 & 0.94 & -1.01 \\
\hline Raceme length $(\mathrm{cm})$ & 12.60 & 11.86 & 0.74 & 0.79 & 12.24 & 12.30 & 0.05 & -0.07 \\
\hline Racemes plant $^{-1}$ & 8.14 & 8.31 & 0.17 & -0.38 & 8.25 & 8.64 & 0.39 & -0.89 \\
\hline Fresh pods plant $^{-1}$ & 28.68 & 23.23 & 5.45 & 1.32 & 31.21 & 29.94 & 1.27 & 0.41 \\
\hline Fresh pod yield plant $^{-1}(\mathrm{~g})$ & 48.77 & 41.58 & 7.18 & 1.08 & 46.74 & 48.03 & 1.29 & -0.24 \\
\hline Fresh seed yield plant $^{-1}(\mathrm{~g})$ & 24.15 & 16.87 & 7.27 & $2.49 * *$ & 26.05 & 23.85 & 2.20 & 0.77 \\
\hline Dry seed yield plant $^{-1}(\mathrm{~g})$ & 13.05 & 8.86 & 4.19 & $2.20 * *$ & 12.43 & 11.67 & 0.76 & 0.63 \\
\hline
\end{tabular}

$*=$ Significant at $\mathrm{P}=0.05 * *=$ Significant at $\mathrm{P}=0.01$ 
Table.10 Comparative quantitative trait means of extra early maturity group (40-50 DAS)

Determinate and indeterminate HACPI6-derived RILs

\begin{tabular}{|c|c|c|c|c|c|c|c|c|}
\hline & \multicolumn{2}{|c|}{2014} & \multirow[b]{4}{*}{ Difference } & \multirow[b]{4}{*}{ t-statistic } & \multicolumn{2}{|c|}{2015} & \multirow[b]{4}{*}{ Difference } & \multirow[b]{4}{*}{ t-statistic } \\
\hline & Determinate & Indeterminate & & & Determinate & Indeterminate & & \\
\hline Number of RILs & 10 & 12 & & & 15 & 22 & & \\
\hline Trait & & & & & & & & \\
\hline Days to $50 \%$ flowering & 46.80 & 45.50 & 1.30 & 1.03 & 47.00 & 48.05 & 1.05 & -1.18 \\
\hline Raceme bearing branches plant $^{-1}$ & 8.52 & 9.55 & 1.03 & -0.57 & 13.24 & 13.28 & 0.04 & -0.05 \\
\hline Raceme length $(\mathrm{cm})$ & 13.38 & 11.80 & 1.58 & $1.69 *$ & 13.14 & 12.45 & 0.69 & 1.52 \\
\hline Racemes plant $^{-1}$ & 9.26 & 8.65 & 0.61 & 0.93 & 9.25 & 9.16 & 0.09 & 0.28 \\
\hline Fresh pods plant $^{-1}$ & 28.40 & 22.75 & 5.65 & $1.68 *$ & 30.93 & 30.59 & 0.34 & 0.16 \\
\hline Fresh pod yield plant $^{-1}(\mathrm{~g})$ & 38.20 & 34.79 & 3.41 & 0.65 & 46.48 & 43.15 & 3.33 & 0.85 \\
\hline Fresh seed yield plant $^{-1}(\mathrm{~g})$ & 17.65 & 16.86 & 0.80 & 0.30 & 22.43 & 21.70 & 0.73 & 0.38 \\
\hline Dry seed yield plant $^{-1}(\mathrm{~g})$ & 9.64 & 8.58 & 1.05 & 0.58 & 11.94 & 10.69 & 1.25 & 1.33 \\
\hline
\end{tabular}

$*=$ Significant at $\mathrm{P}=0.05 * *=$ Significant at $\mathrm{P}=0.01$

Table.11 Comparative quantitative trait means of early maturity group (51-60 DAS) Determinate and indeterminate HACPI -6-dervied RILs

\begin{tabular}{|c|c|c|c|c|c|c|c|c|}
\hline & \multicolumn{2}{|c|}{2014} & \multirow[b]{4}{*}{ Difference } & \multirow[b]{4}{*}{ t-statistic } & \multicolumn{2}{|c|}{2015} & \multirow[b]{4}{*}{ Difference } & \multirow[b]{4}{*}{ t-statistic } \\
\hline & Determinate & Indeterminate & & & Determinate & Indeterminate & & \\
\hline Number of RILs & 04 & 14 & & & 05 & 09 & & \\
\hline Trait & & & & & & & & \\
\hline Days to $50 \%$ flowering & 56.75 & 57.29 & 0.54 & -0.53 & 56.00 & 52.89 & 3.11 & $2.32 *$ \\
\hline Raceme bearing branches plant $^{-1}$ & 6.15 & 9.33 & 3.18 & $-2.78 * *$ & 12.64 & 16.58 & 3.94 & $-2.38 * *$ \\
\hline Raceme length $(\mathrm{cm})$ & 12.785 & 11.19 & 1.60 & 0.79 & 13.25 & 11.39 & 1.86 & $2.62 * *$ \\
\hline Racemes plant $^{-1}$ & 8.35 & 8.30 & 0.05 & 0.04 & 9.24 & 8.67 & 0.57 & 1.09 \\
\hline Fresh pods plant $^{-1}$ & 21.5 & 21.00 & 0.50 & 0.12 & 29.40 & 35.22 & 5.82 & -1.95 \\
\hline Fresh pod yield plant $^{-1}(\mathrm{~g})$ & 30.35 & 28.87 & 1.48 & 0.49 & 45.46 & 45.16 & 0.30 & 0.05 \\
\hline Fresh seed yield plant $^{-1}(\mathrm{~g})$ & 13.6825 & 13.37 & 0.31 & 0.25 & 22.66 & 23.09 & 0.43 & -0.17 \\
\hline Dry seed yield plant $^{-1}(\mathrm{~g})$ & 6.291 & 6.81 & 0.52 & -0.43 & 11.63 & 10.20 & 1.43 & 1.21 \\
\hline
\end{tabular}

\footnotetext{
$*=$ Significant at $\mathrm{P}=0.05 * *=$ Significant at $\mathrm{P}=0.01$
} 
Table.12 Comparative quantitative trait means of medium maturity group (61-75 DAS)

Determinate and indeterminate HACPI 6-derived RILs

\begin{tabular}{|c|c|c|c|c|c|c|c|c|}
\hline & \multicolumn{2}{|c|}{2014} & \multirow[b]{4}{*}{ Difference } & \multirow[b]{4}{*}{ t-statistic } & \multicolumn{2}{|c|}{2015} & \multirow[b]{4}{*}{ Difference } & \multirow[b]{4}{*}{ t-statistic } \\
\hline & Determinate & Indeterminate & & & Determinate & Indeterminate & & \\
\hline Number of RILs & 15 & 32 & & & 08 & 39 & & \\
\hline Trait & & & & & & & & \\
\hline Days to $50 \%$ flowering & 64.00 & 66.75 & 2.75 & $-3.43 * *$ & 68.13 & 67.90 & 0.23 & 0.13 \\
\hline Raceme bearing branches plant $^{-1}$ & 9.76 & 11.56 & 1.80 & -1.11 & 15.05 & 14.05 & 1.00 & 0.6 \\
\hline Raceme length $(\mathrm{cm})$ & 12.36 & 12.35 & 0.01 & 0.01 & 12.68 & 12.71 & 0.03 & -0.04 \\
\hline Racemes plant $^{-1}$ & 8.52 & 8.71 & 0.19 & -0.32 & 9.00 & 9.10 & 0.10 & -0.28 \\
\hline Fresh pods plant $^{-1}$ & 25.13 & 25.50 & 0.37 & -0.14 & 35.88 & 28.51 & 7.36 & $2.59 * *$ \\
\hline Fresh pod yield plant $^{-1}(\mathrm{~g})$ & 33.87 & 35.06 & 1.19 & -0.36 & 43.64 & 41.50 & 2.14 & 0.55 \\
\hline Fresh seed yield plant $^{-1}(\mathrm{~g})$ & 13.99 & 16.39 & 2.40 & -1.5 & 21.16 & 19.66 & 1.50 & 0.75 \\
\hline Dry seed yield plant $^{-1}(\mathrm{~g})$ & 7.71 & 8.01 & 0.30 & -0.26 & 11.37 & 9.82 & 1.55 & 1.61 \\
\hline
\end{tabular}

Table.13 Comparative quantitative trait means of late maturity group (76-90 DAS)

Determinate and indeterminate HACPI 6-derived RILs

\begin{tabular}{|c|c|c|c|c|c|c|c|c|}
\hline & \multicolumn{2}{|c|}{2014} & \multirow[b]{4}{*}{ Difference } & \multirow[b]{4}{*}{ t-statistic } & \multicolumn{2}{|c|}{2015} & \multirow[b]{4}{*}{ Difference } & \multirow[b]{4}{*}{ t-statistic } \\
\hline & Determinate & Indeterminate & & & Determinate & Indeterminate & & \\
\hline Number of RILs $\longrightarrow$ & $\mathbf{0 3}$ & 19 & & & 04 & 15 & & \\
\hline Trait & & & & & & & & \\
\hline Days to $50 \%$ flowering & 86.67 & 88.74 & 2.07 & $-2.11 *$ & 80.75 & 77.27 & 3.48 & 1.78 \\
\hline Raceme bearing branches plant $^{-1}$ & 8.07 & 14.66 & 6.60 & $-3.66 * *$ & 14.95 & 11.53 & 3.42 & $3.08 * *$ \\
\hline Raceme length $(\mathrm{cm})$ & 11.57 & 12.23 & 0.66 & -0.46 & 13.13 & 12.22 & 0.91 & 1.67 \\
\hline Racemes plant $^{-1}$ & 8.13 & 8.72 & 0.58 & -0.62 & 9.15 & 8.71 & 0.44 & 0.8 \\
\hline Fresh pods plant $^{-1}$ & 24.67 & 29.68 & 5.02 & -1.06 & 38.00 & 28.27 & 9.73 & 1.44 \\
\hline Fresh pod yield plant $^{-1}(\mathrm{~g})$ & 35.39 & 44.38 & 9.00 & -0.91 & 50.63 & 42.11 & 8.52 & 0.86 \\
\hline Fresh seed yield plant $^{-1}(\mathrm{~g})$ & 15.06 & 19.07 & 4.01 & -1.05 & 25.28 & 19.77 & 5.51 & 1.37 \\
\hline Dry seed yield plant $^{-1}(\mathrm{~g})$ & 9.96 & 13.03 & 3.07 & -0.61 & 13.95 & 9.03 & 4.91 & $2.02 *$ \\
\hline
\end{tabular}

*= Significant at $\mathrm{P}=0.05 * *=$ Significant at $\mathrm{P}=0.01$ 
Table.14 Estimates of rank correlation between quantitative trait means of HACPI 6-dervied RILs derived evaluated in 2014 and 2015

\begin{tabular}{|c|c|c|c|c|c|c|c|c|}
\hline \multirow[t]{2}{*}{ Trait } & \multicolumn{2}{|c|}{$\begin{array}{c}\text { Extra Early maturity } \\
\text { group }\end{array}$} & \multicolumn{2}{|c|}{ Early maturity group } & \multicolumn{2}{|c|}{ Medium maturity group } & \multicolumn{2}{|c|}{ Late maturity group } \\
\hline & $\begin{array}{c}\text { Determinat } \\
\text { e }\end{array}$ & Indeterminate & $\begin{array}{c}\text { Determinat } \\
\mathrm{e}\end{array}$ & Indeterminate & $\begin{array}{c}\text { Determinat } \\
\text { e }\end{array}$ & Indeterminate & $\begin{array}{c}\text { Determinat } \\
\mathrm{e}\end{array}$ & Indeterminate \\
\hline Days to $50 \%$ flowering & -0.01 & -0.27 & 0.30 & 0.45 & $0.87 *$ & -0.27 & 0.87 & 0.02 \\
\hline Raceme bearing branches plant ${ }^{-1}$ & 0.23 & 0.60 & -0.60 & -0.43 & 0.00 & 0.41 & 0.50 & 0.31 \\
\hline Raceme length $(\mathrm{cm})$ & $0.90 * *$ & -0.10 & -0.40 & -0.10 & 0.10 & $0.61 * *$ & 0.00 & 0.42 \\
\hline Racemes plant $^{-1}$ & 0.43 & 0.60 & 0.30 & -0.23 & -0.45 & $0.66^{* *}$ & 0.00 & -0.01 \\
\hline Fresh pods plant $^{-1}$ & 0.17 & 0.60 & 0.05 & 0.27 & 0.50 & 0.12 & 0.87 & 0.12 \\
\hline Fresh pod yield plant $^{-1}(\mathrm{~g})$ & 0.55 & $0.90 *$ & 0.17 & -0.05 & 0.10 & $0.54 *$ & 0.00 & -0.16 \\
\hline Fresh seed yield plant $^{-1}(\mathrm{~g})$ & 0.05 & -0.40 & $0.90 *$ & -0.23 & $0.80 *$ & $0.48 *$ & 0.00 & -0.12 \\
\hline Dry seed yield plant ${ }^{-1}(\mathrm{~g})$ & 0.32 & 0.10 & 0.20 & -0.10 & 0.20 & $0.72 * *$ & -0.50 & 0.007 \\
\hline
\end{tabular}


HACPI 3-derived indeterminate RILs of early maturity group and determinate RILs of medium maturity group (Table 7) and HACPI 6-derived indeterminate RILs of medium maturity group (Table 14) manifested performance consistency across two years of evaluation for productivity per se traits such as fresh pods plant ${ }^{-1}$, fresh pod yield plant ${ }^{-1}$ and fresh seed yield plant $^{-1}$ as indicated by significant rank correlation coefficient. Both determinate and indeterminate HACPI 6derived RILs of extra early, early and late maturity groups exhibited in-consistent performance across two years of evaluation. These results suggested lack of any definite trend in favour of either determinate or indeterminate RILs of any maturity group with respect to either per se performance or consistency of performance for any of the traits investigated. Thus, our results provided ample evidence for possibility of fixing the loci controlling economic traits in the genetic background of both determinate and indeterminate varieties irrespective of their maturity duration. To the best of our knowledge, the present results are based on a large number of determinate and indeterminate RILs with a range of maturity duration and comparable genetic background and variation for the traits for which the genotypes are compared.

\section{Acknowledgement}

Senior author gratefully acknowledges Krikhouse Trust, UK providing financial support in the form of fellowship for conducting thesis research for partial fulfillment for the award of Ph.D. degree by university of agricultural sciences, Bengaluru, India.

\section{References}

Adams, M. W. 1982. Plant architecture and yield breeding in Phaseolus vulgaris,
Iowa State Journal of Research. 56: 225-254.

Ayyangar, G. N. R., and K. K. K. Nambiar. 1935. Studied in Dolichos lablab (Roxb.) (L.). The Indian field and garden bean. Proceedings of Indian Academy of Sciences. 1(12): 857-867.

Bernard, R. L. 1972. Two genes affecting stem termination in soybeans, Crop Science. 12: 235-239.

Byregowda, M., G. Gireesh, S. Ramesh, P. Mahadevu, C. M. Keerthi.2015.Descriptors of Dolichos bean (Lablab purpureus L.). Journal of Food Legumes. 28 (3): 203-214.

Chang, J. F., D. E. Green, and R. Shibles. 1982. Yield and agronomic performance of semi-determinate and indeterminate soybean stem types. Crop Science. 22: 97- 101.

Cober, E. R., and J. W. Tanner. 1995. Performance of related indeterminate and tall determinate soybean lines in short-season areas. Crop Science. 35: 361-364.

Cooper, R. L., and A. Waranyawat. 1985. Effect of three genes ( $p d$, Rps 1 and ln) on plant height, lodging and seed yield in indeterminate and determinate nearisogenic lines of soybeans. Crop Science. 25:90-92.

Federer, W. T. 1956. Augmented (or hoonuiaku) designs. Hawaii Plant Research. 2:191-208.

Foley, T. C., J. H. Orf, and J. W. Lambert. 1986. Performance of related determinate and indeterminate soybean lines. Crop Science. 26: 5-8.

Fuller, D.Q. 2003. African crops in prehistoric South Asia: a critical review. In: Food, fuel, fields-progress in African archaebotany, Heinrich-BarthInstitute, Koln, Germany, Africa Prehistorica. 15: 239271.

Huyghe, C. 1998. Genetics and genetic modifications of plant architecture in 
grain legumes: a review. Agronomie. 18 (5-6): 383-411.

Julier, B., C. Huyghe, and J. Papineau. 1993a. Dry matter and nitrogen accumulation and seed yield in determinate autumnsown white lupins (Lupinusalbus L.). Agronomie. 13: 877-888.

Julier, B., C. Huyghe, J. Papineau, G. F. J. Milford, J. M. Day, C. Billot, and P. Mangin. 1993b. Yield and yield stability of determinate and indeterminate autumn-sown white lupins (Lupinusalbus L.) grown at different locations in France and the UK. Journal of Agriculture Science. 121: 177-186.

Karivaratharaju, T. V., and K. R. Ramamoorthy. 1990. Photosynthesis by fruit walls of lablab bean and their role in seed development. Indian Journal of Plant Physiology. 33(4): 351-354.

Kato, S., K, Fujii, S. Yumoto, M. Tshimoto, T. Shiraiwa, T. Sayama, A. Kikuchhi, and T. Nishio. 2015. Seed yield and its components of indeterminate and determinate lines in recombinant inbreed lines of soybean. Breeding Science. 65: 154-160.

Kay, D.E. 1979. Hyacinth Bean - Food Legumes, Crop and Product Digest No.3. Tropical Products Institute., xvi: 184-196.

Keerthi, C. M., S. Ramesh, M. Byregowada, A. Mohan Rao, P. V. Vaijayanthi, N. Chandrakanth, and M. S. Shivakumar. 2016. High yielding Vs. Low yielding testers to identify advanced breeding lines for general combining ability in dolichos bean (Lablab purpureus L.). Journal of Crop Improvement.30 (1):95-106.

Keerthi, C. M., S. Ramesh, M. Byregowda, A. M. Rao, B. S. Rajendra Prasad, and P. V. Vaijayanthi. 2014a. Genetics of growth habit and photoperiodic response to flowering time in dolichos bean (Lablab purpureus (L.) Sweet).Journal of Genetics. 93: 203206.

Keerthi, C. M., S. Ramesh, M. Byregowda, A. Mohan Rao, G. A. Mary Reena, and N. Chandrakant. 2014b. Performance stability of photoperiod sensitive vs. insensitive dolichos bean (Lablab purpureus L. var. Lignosus) cultivars under delayed sowing conditions. Australian Journal of Crop Science. 8(12): 1658-1662.

Kelly, J. D., M. W. Adams, and G. V. Varner. 1987. Yield stability of determinate and indeterminate dry bean cultivars, Theor. Appl. Genetics. 74: 516-521.

Kim, S. E., H. Okubo, and Y. Kodama. 1992. Growth response of dwarf Lablab bean [Lablab purpureus (L.) Sweet] to sowing date and photoperiod.Journal of Japan Society Horticultural Science. 61(3): 589-594.

Koscielniak, J., W. Filek, and G. Augustyniak. 1990. Photosynthetic activity in different organs of field beans (Viciafaba L. minor) with indeterminate and determinate growth habit. Acta Physiology Plant. 12: 95103.

Nadal, S., A. Cabello, F. Flores, and M. J. Moreno. 2005. Effect of growth habit on agronomic characters in faba bean. Agriculture conspectus scientificus. 70(2): 43-47.

Nleya, T. M., A. E. Slinkard, and A. Vandenberg. 1999. Evaluation of determinate and indeterminate pinto bean cultivars under an available soil moisture gradient. Cannadian Journal of Plant Science. 79: 27-34.

Patterson, H. D., and R. Thompson. 1971. Recovery of inter-block information when block sizes are unequal. Biometrika. 58(3): 545-554.

Pervez, A. Q., F. P. Gardner, and K. J. Boote. 1989. Determinate and indeterminate- 
type soybean cultivar responses to pattern, density and planting date. Crop Science. 29:150-157.

Purseglove, J.W. 1968. Tropical Crops, Dicotyledons, Vol L London, UK; Longmans Greens and Company Ltd. pp. 273-276.

Robinson, S. L., and J. R. Wilcox. 1998. Comparison of determinate and indeterminate soybean near-isolines and their response to row spacing and planting dates. Crop Science. 38(6): 1554-1557.

Sheoran, I. S., H. R. Singal, and R. Singh. 1987. Photosynthetic characteristics of chickpea (Cicer arietinum L.) pod wall during seed development. Indian Journal of Experimental Biology. 25: 843-847.

Shivashankar, G., R. S. and Kulkarni. 1989. Field bean [Dolichos lablab (L.)], var. lignosusPrain. Indian Hort. 34: 24-27.

Singh, V. P., and G. Schroeder. 1988. Effect of stem termination on some metric traits in faba bean. Fabis Newsletter. 20:19-21.

Skerman, P.J., D.G. Cameron, and F. Riveros.
1991. Leguminosasforrajerastropicales. Colección FAO: Producción y Protección Vegetal, No. 2. Organización de lasNacionesUnidaspara la Agricultura y la Alimentación.Roma. 5, Academic Press, New York, pp. 609635.

Vishwanath, S. R., G. Shivashankar, and A. Manjunath. 1971. Non season-bound Dolichos lablab with new plant type. Current Science. 40: 667-668.

White, J. W., J. Kornegay, J. Castillo, C. H. Molano, C. Cajiao, and G. Tejada. 1992. Effect of growth habit on yield of large-seeded bush cultivars of common bean. Field Crops Research. 29:151161.

Whyte, R.O., G. Nilsson-Leissner, and H.C. Trumble, 1953.Legumes in agriculture, Rome Italy FAO, pp.272-273.

Wicox, J. R., and G. D. Zhang. 1997. Relationship between seed yield and seed protein in determinate and indeterminate soybean populations. Crop Science. 37:361-364.

\section{How to cite this article:}

Uday Kumar, H.R., M. Byre Gowda and Ramesh, S. 2017. Does Relative Performance of Determinate and Indeterminate Dolichos Bean (Lablab purpureus L. Sweet) Recombinant Inbred Lines (RILs) Depend on Maturity Duration? Int.J.Curr.Microbiol.App.Sci. 6(7): 527540. doi: https://doi.org/10.20546/ijcmas.2017.607.064 